\title{
ANALYTICAL PREDICTIONS OF THE AIR GAP RESPONSE OF FLOATING STRUCTURES
}

\author{
Lance Manuel $^{1}$, Bert Sweetman ${ }^{2}$ and Steven R. Winterstein ${ }^{2}$ \\ ${ }^{1}$ Department of Civil Engineering, University of Texas at Austin, TX 78712 \\ ${ }^{2}$ Department of Civil and Environmental Engineering, Stanford University, CA 94305
}

\begin{abstract}
Two separate studies are presented here that deal with analytical predictions of the air gap for floating structures. (1) To obtain an understanding of the importance of first- and second-order incident and diffracted wave effects as well as to determine the influence of the structure's motions on the instantaneous air gap, statistics of the air gap response are studied under various modeling assumptions. For these detailed studies, a single field point is studied here - one at the geometric center (in plan) of the Troll semi-submersible.

(2) A comparison of the air gap at different locations is studied by examining response statistics at different field points for the semi-submersible. These include locations close to columns of the four-columned semi-submersible. Analytical predictions, including first- and second-order diffracted wave effects, are compared with wave tank measurements at several locations. In particular, the gross root-mean-square response and the 3-hour extreme response are compared.
\end{abstract}

\section{BACKGROUND}

The air gap response, and potential deck impact, of ocean structures under random waves is generally of considerable interest. While air gap modeling is of interest both for fixed and floating structures, it is particularly complicated in the case of floaters because of their large volume, and the resulting effects of wave diffraction and radiation. These give rise to two distinct effects: (1) global forces and resulting motions are significantly affected by diffraction effects; and (2) local wave elevation modeling can also be considerably influenced by diffraction, particularly at locations above a pontoon and/or near a major column. Both effects are important in air gap prediction: we need to know how high the wave rise (step 2), and how low the deck translates vertically (due to net heave and pitch) at a given point to meet the waves. Moreover, effects (1) and (2) are correlated in time, as they result from the same underlying incident wave excitation process.

We focus here on analytical diffraction models of air gap response, and its resulting stochastic nature and numerical predictions under random wave excitation. Attention is focused on a semi-submersible platform, for which both slow-drift motions (heave/pitch) and diffraction effects are potentially significant. This air gap response presents several new and interesting challenges. It is the first response limit state where we need to simultaneously include both second-order sum- and difference-frequency effects (on the wave surface), and second-order differencefrequency effects (on slow drift motions and generally, on the wave surface as well). The sum- and differencefrequency waves and the difference-frequency heave and pitch motions can both influence the air gap. The air gap response is further complicated because the heave, pitch, and roll motions of the floating structure are generally coupled. Moreover, the motions and the net wave elevation, both of which affect the air gap, are correlated in time as they result from the same underlying incident wave excitation process. Note that air gap modeling has been the subject of previous work within the Reliability of Marine Structures Program at Stanford University. For example, Winterstein and Sweetman (1999) apply a fractile-based approach to develop a scaling factor between the statistics of the incident waves and those of the associated air gap demand.

Results are shown here from frequency-domain analyses which permit careful study and isolation of various effects: e.g., wave forces on a fixed (locked-down) structure, the effect of structural motions on air gap response, and finally, the effect of different local wave elevation models. For reference, a complete second-order diffraction model is formulated and studied. Compared with this complete model, various simplified models are imposed and evaluated: (a) second-order wave elevation effects are neglected completely, or (b) these second-order effects are approximated 
by analytical Stokes theory, which retains second-order effects on the incident wave but neglects second-order diffraction. Use of (a) and (b) would significantly simplify the analysis, avoiding the costly step of second-order diffraction. The local wave modeling in step (b) is found to be quite important in predicting the air gap response of two semi-submersibles studied here.

\section{THEORY}

Volterra Series and Transfer Functions

In modeling nonlinear systems, such as floating structures, it is common to employ Volterra series that permit one to describe the response (output) of such systems. The nonlinear system is defined in terms of first- and second-order transfer functions. For floating structures, these transfer functions are obtained from first- and second-order wave diffraction analysis programs (e.g., WAMIT, 1995).

In order to study the response of a floating structure to random seas, we start by defining an irregular sea surface elevation, $\eta(t)$, as a sum of sinusoidal components at $N$ distinct frequencies:

$$
\begin{aligned}
\eta(t) & =\sum_{k=1}^{N} a_{k} \cos \left(\omega_{k} t+\theta_{k}\right) \\
& =\operatorname{Re} \sum_{k=1}^{N} A_{k} \exp \left(i \omega_{k} t\right) ;
\end{aligned}
$$

where $a_{k}=\sqrt{2 S_{\eta}\left(\omega_{k}\right) \Delta \omega} ; \quad A_{k}=a_{k} \exp \left(i \theta_{k}\right)$

in which $S_{\eta}(\omega)$ represents the wave power spectrum.

Any response quantity, $x(t)$, may be described by a second-order Volterra series representation:

$$
x(t)=x_{1}(t)+x_{2}(t)=x_{1}(t)+x_{2-}(t)+x_{2+}(t)
$$

where $x_{1}(t), x_{2-}(t)$, and $x_{2+}(t)$ are the first-order, second-order difference-frequency and second-order sum-frequency contributions, respectively, to the response. We can write each of these three components in terms of the appropriate (first- and second-order) transfer functions. Thus,

$$
\begin{aligned}
& x_{1}(t)=\operatorname{Re} \sum_{k=1}^{N} A_{k} H_{k}^{(1)} \exp \left(i \omega_{k} t\right) \\
& x_{2-}(t)=\operatorname{Re} \sum_{k=1}^{N} \sum_{l=1}^{N} A_{k} A_{l}^{*} H_{k l}^{(2-)} \exp \left[i\left(\omega_{k}-\omega_{l}\right) t\right] \\
& x_{2+}(t)=\operatorname{Re} \sum_{k=1}^{N} \sum_{l=1}^{N} A_{k} A_{l} H_{k l}^{(2+)} \exp \left[i\left(\omega_{k}+\omega_{l}\right) t\right]
\end{aligned}
$$

In Equation (3), $H_{\mathrm{k}}{ }^{(1)}$ denotes the first-order transfer function, while $H_{\mathrm{k}}^{(2+)}$ and $H_{\mathrm{k}}^{(2-)}$ are the second-order sum- and difference-frequency transfer functions. Also, these transfer functions can describe any quantity of interest such as wave loads, platform motions, forces, etc.

\section{$\underline{\text { Response Moments and Extremes }}$}

In the reliability analysis of offshore structures, response quantities of interest include extremes and fatigue damage. Exact statistics of these quantities are not known even for linear (Gaussian) responses, much less for second-order Volterra models such as we use here. Hence, we must approximate these quantities, using limited response statistics for the total (first- plus second-order) process given by Equations (2) and (3). We choose here to characterize the total response process by its first four statistical moments (mean, standard deviation, skewness, and kurtosis) which are then used to estimate response extremes. These first four statistical moments of the response are defined as 


$$
\begin{aligned}
& m_{x}=E[x(t)] \\
& \sigma_{x}^{2}=E\left[\left(x(t)-m_{x}\right)^{2}\right] \\
& \alpha_{3 x}=E\left[\left(x(t)-m_{x}\right)^{3}\right] / \sigma_{x}^{3} \\
& \alpha_{4 x}=E\left[\left(x(t)-m_{x}\right)^{4}\right] / \sigma_{x}^{4}
\end{aligned}
$$

In order to obtain moments of the response process given in Equations (2) and (3), it is convenient to rewrite $x(t)$ in terms of standard Gaussian processes, $u_{j}(t)$. In general, whether second-order diffraction effects are included or neglected, the first- and second-order processes, $x_{1}(t)$ and $x_{2}(t)$, can be rewritten in terms of the standard Gaussian processes, $u_{j}(t)$ :

$$
\begin{aligned}
& x_{1}(t)=\sum_{j=1}^{2 N} c_{j} u_{j}(t) \\
& x_{2}(t)=\sum_{j=1}^{2 N} \lambda_{j} u_{j}^{2}(t)
\end{aligned}
$$

in which $N$ is the number of frequency components of $x_{I}(t)$. The coefficients $c_{j}$ and $\lambda_{j}$ are obtained by solving an eigenvalue problem of size $N$ for problems involving difference or sum frequencies only (Naess, 1986, Naess, 1992), or of size $2 N$ for problems - such as these air gap response problems - which involve both (e.g., Winterstein et al, 1994). The resulting moments of $x$ can be found directly from the coefficients $c_{j}$ and $\lambda_{j}$ in Equation (5):

$$
\begin{aligned}
& m_{x}=\sum_{j=1}^{2 N} \lambda_{j} \\
& \sigma_{x}^{2}=\sum_{j=1}^{2 N}\left(c_{j}^{2}+\lambda_{j}^{2}\right) \\
& \alpha_{3 x}=\frac{1}{\sigma_{x}^{3}} \sum_{j=1}^{2 N}\left(6 c_{j}^{2} \lambda_{j}+8 \lambda_{j}^{3}\right) \\
& \alpha_{4 x}=3+\frac{1}{\sigma_{x}^{4}} \sum_{j=1}^{2 N}\left(48 c_{j}^{2} \lambda_{j}^{2}+48 \lambda_{j}^{4}\right)
\end{aligned}
$$

Once these response moments are found, the response process $x(t)$ is assumed to be related to a standard Gaussian process $u(t)$ using a Hermite transformation model (Winterstein, 1988):

$$
x(t)=g(u(t))=m_{x}+\kappa \sigma_{x}\left[u(t)+c_{3 \mathrm{H}}\left((u(t))^{2}-1\right)+c_{4 \mathrm{H}}\left((u(t))^{3}-3 u(t)\right)\right]
$$

in which $c_{3 \mathrm{H}}$ and $c_{4 \mathrm{H}}$ are chosen to preserve the skewness and kurtosis of $x(t)$, and $\kappa=\left(1+2 c_{3 \mathrm{H}}^{2}+6 c_{4 \mathrm{H}}^{2}\right)^{-1 / 2}$ is chosen to preserve the standard deviation of $x(t)$.

The key assumption of Equation (7) is that it relates the actual non-Gaussian response, $x(t)$, to a standard Gaussian response, $u(t)$, at each point in time. It follows that for any realizations of these processes over time $T$, their respective extreme values - denoted $X_{\max , \mathrm{T}}$ and $U_{\max , \mathrm{T}}$ - are related by the same functional transformation, $g$ :

$$
X_{\max , \mathrm{T}}=g\left(U_{\max , \mathrm{T}}\right)
$$

(Note that in practice, the cubic transformation $g$ is constrained to remain monotonic, so that extrema of $u(t)$ and $x(t)$ are ensured to map directly.)

Finally, since Equation (7a) applies to each realization of $X_{\max , \mathrm{T}}$ and $U_{\max , \mathrm{T}}$, the same relation must also apply to their $p$-fractile values, denoted $x_{\max , \mathrm{p}}$ and $u_{\max }$ T, : 


$$
x_{\max , \mathrm{p}}=g\left(u_{\max , \mathrm{p}}\right)
$$

It only remains to use standard results for Gaussian process theory to estimate $u_{\max } \mathrm{T}, \mathrm{p}$. With the assumption of Poisson upcrossings (Crandall and Mark, 1963),

$$
P\left[U_{\max , \mathrm{T}}<u\right]=\exp \left[-v_{0} T \exp \left(-\frac{u^{2}}{2}\right)\right]
$$

Setting this probability to $p$ and solving for $u$, the corresponding $p$-fractile is found to be

$$
u_{\max , \mathrm{p}}=\left[2 \ln \left(\frac{v_{0} T}{\ln (1 / p)}\right)\right]^{1 / 2}
$$

where $v_{0}$ is the average rate at which $u(t)$, and hence, $x(t)$, upcross their median values. Hence, the Hermite response estimate of extreme statistics - specifically, arbitrary fractiles of the maximum value in duration $T$ - are found from Equation (7), using for $u(t)$ the corresponding Gaussian extreme fractile from Equation (8).

\section{Air Gap Response}

In order to determine the instantaneous air gap, it is useful first to consider the net wave elevation, $\eta_{\text {NET }}(t)$, with respect to a fixed origin. The logical next step is to consider the relative wave elevation, $r(t)$, measured with respect to the moving structure:

$$
r(t)=\eta_{N E T}(t)-\delta(t)
$$

At the field point of interest, $(x, y), \delta(t)$ denotes the net vertical displacement, which in turn is generally given in terms of the heave $\left(\xi_{3}\right)$, roll $\left(\xi_{4}\right)$, and pitch $\left(\xi_{5}\right)$ motions:

$$
\delta(t)=\xi_{3}(t)+y \cdot \xi_{4}(t)-x \cdot \xi_{5}(t)
$$

The available air gap $a(t)$ is the difference between the still-water air gap, $a_{0}$, and the relative wave, $r(t)$ :

$$
a(t)=a_{0}-r(t)
$$

In Figure 1, a schematic diagram is shown where various quantities defined in Equations (9) to (11) needed to define the air gap response are indicated.

The instantaneous net wave elevation, $\eta_{N E T}(t)$, in Equation (9) is a result of both the incident waves that would occur if the structure were not present, and the diffracted waves that arise because of the presence of the structure that alters the flow field:

$$
\begin{aligned}
\eta_{N E T}(t) & =\eta_{1, N E T}(t)+\eta_{2, N E T}(t) \\
\eta_{1, N E T}(t) & =\eta_{1, I}(t)+\eta_{1, D}(t) \\
\eta_{2, N E T}(t) & =\eta_{2, I}(t)+\eta_{2, D}(t)
\end{aligned}
$$

In Equation (12), we see that the net wave elevation is made up of first- and second-order waves; moreover, individually the incident waves (subscript: $I$ ) and the diffracted waves (subscript: $D$ ) will contribute to the first- and second-order waves. The first-order incident wave $\eta_{l, I}$ is modeled here as a stationary Gaussian process, and consistent values of $\eta_{1, D}, \eta_{2, I}$, and $\eta_{2, D}$ are calculated from hydrodynamic theory. Note that this second-order diffraction term is likely to be the most expensive quantity to calculate within the numerical, boundary-value 
diffraction analysis. It is also perhaps the quantity most subject to questions of adequate modeling, meshing, etc. Therefore, we shall study here the adequacy of a "hybrid" model that omits $\eta_{2, D}$; i.e., retaining incident wave effects to second order but diffraction corrections to first order only.

In Equation (12), the first-order incident wave, $\eta_{1, l}$, is the Gaussian input wave process, which was denoted $\eta(t)$ for simplicity in Equation (1). Also, we approximate the spectrum of $\eta_{1, I}$ by that of the total incident wave, $\eta_{l, I}+\eta_{2, I}$. Differences between these spectra, due to second-order Stokes contributions, have been found to be generally negligible.

Application of Methodology to Floating Structures

The methodology using Volterra series models to describe the response of floating structures and the moment-based extreme estimation has been implemented in a post-processing analysis routine, TFPOP (Ude et al., 1996). This routine uses first- and second-order force transfer functions (from force calculations involving diffraction analysis) as well as added mass and damping (from first-order wave radiation). TFPOP combines these load transfer functions together with stiffness, damping, and inertia properties of the structure and, thus, constructs first- and second-order transfer functions to any response quantity, $x(t)$. In general, an analysis can include forces/moments in any of six degrees of freedom and can be used to estimate response quantities such as motions, accelerations, tether tensions, etc. The implementation and its theoretical basis (second-order model with moment-based extremes and fatigue estimation) have been verified in several previous studies. The different structures analyzed include TLP's, semi-submersibles, and spar buoys. Cases studied included TLP responses that were sensitive to second-order highfrequency loads (e.g., tether fatigue and heave acceleration - see Winterstein et al., 1994) as well as slow drift motions that were influenced by second-order low-frequency loads (e.g., surge motions of a TLP (Winterstein et al., 1994) and of a semi-submersible and a spar buoy (Winterstein et al., 1999)). The first reference (Winterstein et al., 1994) verifies the accuracy of the Hermite model, for both sum- and difference-frequency applications, through detailed comparison with simulation. An associated study (Jha et al., 1997) shows the ability of these second-order models, with Newman's approximation, to accurately predict both slow-drift and wave-frequency motions found from spar buoy model tests.

The air gap response, as described by Equations (9) through (12) above, has been analyzed in an extended, air gapspecific analysis methodology, based on second-order Volterra series and Hermite extreme models (Manuel and Winterstein, 1998). This methodology permits one to selectively include or exclude the various contributions to the net wave elevation in Equation (12). Thus, one can study the relative influences of first- versus second-order waves, and of incident versus diffracted waves.

\section{PLATFORM DESCRIPTION}

The structure chosen for the numerical studies is the Troll concrete semi-submersible platform. Figure 2 shows a plan view of the platform that has four columns and plan dimensions, $100 \mathrm{~m} \mathrm{X} 100 \mathrm{~m}$. The still-water air gap is 25 meters and the mean water depth is 325 meters. Wave tank measurements of air gap were made at seven different field point locations. At these same locations, WAMIT diffraction analyses were performed for waves with different headings and with wave periods ranging from 7.4 to 20.0 seconds. In the following, we discuss comparison of the air gap response statistics at three field point locations indicated by the numbers 1,5 , and 6 in Figure 2.

\section{RESULTS I: PARAMETRIC STUDY AT MID-POINT LOCATION}

We first consider analytical predictions of the relative wave elevation for the Troll semi-submersible. We are interested here in predicting the influence of alternative modeling options in describing the relative wave. For the sake of this exercise, we will study the air gap at a location directly below the center of the platform. This is field point no. 1 in Figure 2. We consider a single seastate of 3 hours duration that is characterized by head seas (zerodegree heading) with a significant wave height of 10.6 meters and a spectral peak period of 12.5 seconds. This is a seastate for which model tests are available for comparison. 
To isolate the effects of incident and diffracted waves of first- and second-order, and the effect of net vertical motion, we consider the following cases:

Case 0 Only first-order incident waves $\left(\eta_{1, I}\right)$ included; structure locked down (i.e., motions prevented, $\delta=0$ ).

Case 1 Only first- and second-order incident waves $\left(\eta_{1, I}\right.$ and $\left.\eta_{2, I}\right)$ included; structure locked down.

Case 2 First-order incident and diffracted waves $\left(\eta_{1, I}\right.$ and $\left.\eta_{1, D}\right)$, second-order sum- and difference-frequency effects on the incident waves $\left(\eta_{2, I}\right)$ included; structure locked down. This is the "hybrid" model (incident waves correct to second order; diffraction corrections to first order) referred to above.

Case 3 Full first- and second-order incident and diffracted waves $\left(\eta_{1, I}, \eta_{1, D}, \eta_{2, I}\right.$, and $\left.\eta_{2, D}\right)$ included; structure locked down. Note that second-order diffraction results are available here for sum-frequency effects only. Accordingly, the difference-frequency transfer function for wave elevation is again estimated by Stokes theory (ignoring the presence of the structure) as in Case 2. From experience with other cases, we anticipate the neglect of diffraction effects on difference-frequency terms to have relatively minor consequence, although their inclusion will typically serve to mildly decrease nonlinear effects.

Heave Structure's heave motion $\left(\xi_{3}\right)$ studied.

Base First- and second-order incident and diffracted waves $\left(\eta_{1, I}, \eta_{1, D}, \eta_{2, I}\right.$ and $\left.\eta_{2, D}\right)$ included; structure permitted to move in heave, pitch, and roll (i.e., $\delta \neq 0$ )

Table 1 summarizes the predicted response statistics for the Troll submersible in each of the cases defined. (Note that all results seek to model the air gap response except "Heave", which reflects only the vertical structural motion.) The statistics for the total response (i.e., including first- and second-order contributions) are presented in Table 1. Also included are response statistics if only the first-order terms are retained.

From Table 1, the following findings may be noted as we move across the various cases:

Case 0 Because Case 0 includes only first-order incident waves, the net wave elevation (TOTAL) is seen to be Gaussian as expected (e.g., skewness $=0$ and kurtosis $=3$ ). Also, the rms response ( 2.65 meters) is equal to one-fourth of the significant wave height (10.6 meters) as expected. The Gaussian character of the response is also confirmed by the peak factor on the median extreme of 3.8, as found from Equation (8) with $p=0.5, T=3$ hours $=10800 \mathrm{sec}$, and response median-upcrossing rate, $v_{0}=0.1 \mathrm{sec}^{-1}$.

Case 1 Case 1 includes a second-order Stokes incident wave process, which causes the net wave elevation to be non-Gaussian and positively skewed. The second-order process provides a small contribution to the total response: the total process rms ( 2.70 meters) is only $2 \%$ larger than that of the first-order process $(2.65$ meters). This suggests that the spectrum of the first-order process is well approximated by that of the total process, as implicitly assumed here. The peak factor of the total process is 4.1 (only slightly nonGaussian).

Case 2 Addition of the first-order diffracted waves in Case 2 (relative to Case 1) has the effect of raising the rms of the first-order process by $23 \%$ and the median extreme by $20 \%$. (Because a linear effect has been added, we would expect roughly proportional increases in rms and extreme levels.) The peak factor of the total process response is 3.7 (i.e., the total process is more Gaussian than in Case 1 due to the larger relative contribution of the first-order effects).

Case 3 Addition of second-order diffracted waves in Case 3 (relative to Case 2) causes a large increase in response, most notably in its extreme levels. In particular, the rms level changes only from 3.27 to 3.50 , while the peak factor grows from 3.7 to 5.5. As we shall see below, these larger peak factors may be more consistent with the observed behavior in model test experiments. Note also that these enhanced peak factors are due to the marked non-Gaussian behavior predicted in this case: the skewness value is predicted to be 0.54 , and the kurtosis value to be 4.12 , both far exceeding corresponding values in all earlier cases.

Heave The results for heave motions again show that nonlinear effects (here, the effect of difference-frequency slow-drift motions) only mildly influence rms values (0.78 increases only to 0.79$)$, but more notably increases skewed, non-Gaussian behavior (skewness value of 0.21 ).

Base Finally, the base case results predict the relative wave response, from a structure now permitted to move. These results combine our "best" model of the wave elevation (including second-order diffraction effects as in Case 3) with our correlated model of associated vertical motions (at this midpoint location, due to heave only). As in Case 3, the results here show particularly strong non-Gaussian behavior: skewness value of 0.54 , kurtosis of 4.55 , and peak factor of 6.1 . In view of their similarity with Case 3 values, these strongly non-Gaussian effects appear due to the presence of second-order diffracted waves, and are not weakened when structural motions are included. Of course, by permitting the structure to move with the waves, the 
relative wave response is reduced as compared with the locked-down structure in Case 3: reductions in standard deviations from $3.50 \mathrm{~m}$ to $2.85 \mathrm{~m}$, and in median extremes from $19.23 \mathrm{~m}$ and $17.18 \mathrm{~m}$. These reductions appear quite consistent with the marginal heave statistics shown in Table 1.

\section{RESULTS II: BEST PREDICTIONS VERSUS OBSERVATIONS AT SELECTED LOCATIONS}

We next study three different field point locations and compare analytical predictions with model test results. Four different seastates are considered:

1. $\quad H_{s}=10.6 \mathrm{~m}, T_{p}=12.5 \mathrm{~s}$

2. $\quad H_{s}=12.6 \mathrm{~m}, T_{p}=13.5 \mathrm{~s}$

3. $\quad H_{s}=13.3 \mathrm{~m}, T_{p}=13.0 \mathrm{~s}$

4. $\quad H_{s}=14.5 \mathrm{~m}, T_{p}=14.0 \mathrm{~s}$

These four seastates are chosen to conform with 3-hour model tests, all performed for head seas. In Table 2, results are shown for three field points, including again the platform mid-point and two other locations nearer to columns. In all cases, we focus on numerical values of the rms and peak factor (PF) only.

We first compare results from two different models: the most detailed model (the earlier "base" case), and its nearest competitor (which neglects second-order diffraction effects on the wave elevation, calculated at each field point). As discussed earlier, this simpler ("hybrid") model is significantly less costly. As such, it is interesting to consider whether it can usefully approximate the more expensive base case results.

Comparing the results of the two models (in Table 2), second-order diffraction is found to (1) only moderately increase the rms response, but (2) markedly increase peak factors and hence predicted extremes. Thus, even if second-order effects in the incident wave are retained, unconservative air gap predictions may result if second-order diffraction effects are neglected. The strength of this second-order diffraction correction is found to depend on the location; particularly strong non-Gaussian behavior is predicted at the platform mid-point, as compared with predictions corresponding to other points nearer a column.

Finally, we compare the model predictions with the observed wave tank statistics, shown in the final two columns of Table 2. We first note that these observed extremes (peak factors) each arise from a single 3-hour test, and are therefore rather noisy estimates of the median values across many similar 3-hour conditions. Even with that caveat, these results strongly suggest that second-order diffraction effects should not be ignored. Models that ignore these effects underestimate the observed peak factors, across all tests and locations considered.

When second-order diffraction effects are included, peak factors are no longer systematically underestimated. Agreement with observations remains imperfect, however. At some locations such as the platform midpoint, the predictions may appear somewhat "too nonlinear." Nonetheless, these results suggest that nonlinear diffraction effects can be important, and should be studied further. We believe that the general statistical models presented here, which estimate extremes from a limited set of statistical moments, offer an efficient approach to assess the impact of various nonlinear models on extreme response levels. To support this, Figure 3 compares predicted model test results (see Winterstein and Sweetman, 1999) from a smaller semi-submersible platform, Veslefrikk for two seastates (with 12 and 14 meter significant wave heights). Results are shown for a locked-down structure across 9 measured field points. Two moment-based Hermite predictions are shown: (1) results using moments from the "hybrid" diffraction model, and (2) results using moments from the measured time histories. As with the Troll results, the hybrid model consistently underestimates the air gap demand. Knowledge of the actual moments generally improves prediction, particularly in the 12 meter seastate. Although accurate results have been found for 20-minute extremes, for the 14 meter seastate, results diverge for the longer 3-hour seastate duration shown. It may be that more than four moments are necessary in this case to capture the behavior of the largest extremes. Physically, in this more severe seastate there may be some change in the wave process, such as minor wave impact with the vessel at locations other than those with air gap probes, which leads to a reduction in the most extreme measured air gap events. 


\section{CONCLUSIONS}

We have presented a methodology for describing the net wave elevation and, hence, the air gap response of floating structures. This methodology has been demonstrated to allow different levels of modeling the net wave elevation, and of modeling the relative wave surface with respect to the moving structure. For example, the second-order diffracted waves may be omitted from the analysis if only a first-order diffraction analysis has been carried out.

The importance of the diffracted first- and second-order waves, relative to their incident wave counterparts, has been studied. We find that for the Troll semi-submersible, a full second-order analysis (including both incident and diffracted waves to second order) is necessary. It is found to be unconservative here to neglect second-order diffracted waves, although this practice is often necessitated by the expense of second-order diffraction analyses.

In comparing results across field point locations, we find important non-Gaussian effects that differ slightly depending on each field point's proximity to a column. A field point at the center of the platform exhibited the greatest non-Gaussian character relative to field points near a column. The non-Gaussian character is largely a result of second-order diffracted waves.

\section{ACKNOWLEDGMENTS}

The authors gratefully acknowledge the support provided by a grant from the Offshore Technology Research Center. Additional support was provided by the sponsors of Stanford University's Reliability of Marine Structures (RMS) program. The authors acknowledge the assistance received from Norsk Hydro, and especially from Gudmund Kleiven for supplying the diffraction analysis results for the Troll semi-submersible.

\section{REFERENCES}

1. Crandall, S.H. and W.D. Mark (1963). "Random Vibration in Mechanical Systems," Academic Press, 1963.

2. Jha, A.K., P.R. De Jong, and S.R. Winterstein (1997). "Motions of a Spar Buoy in Random Seas: Comparing Predictions and Model Test Results" Proceedings, BOSS-97, Vol. 2, pp. 333-347.

3. Manuel, L. and S.R. Winterstein (1998). "Estimation of Air Gap Statistics for Floating Structures: Release of TFPOP Version 2.2 - A Computer Program for Performing Stochastic Response Analysis of Floating Structures," Technical Note TN-4, Reliability of Marine Structures Program, Stanford University.

4. Naess, A. (1986). "The Statistical Distribution of Second-Order Slowly Varying Forces and Motions," Applied Ocean Research, 8, pp. 110-118.

5. Naess, A. (1992). "Prediction of Extremes Related to the Second-Order, Sum-Frequency Responses of a TLP," Proceedings, $2^{\text {nd }}$ International Offshore Polar Engineering, ISOPE. pp. 436-443

6. Ude, T.C., S. Kumar, and S.R. Winterstein (1996). "TFPOP 2.1: Stochastic Response Analysis of Floating Structures under Wind, Current, and Second-Order Wave Loads,” Technical Report RMS-18, Reliability of Marine Structures Program, Stanford University.

7. WAMIT, 4.0 (1995). "WAMIT: A Radiation-Diffraction Panel Program for Wave-Body Interaction - Users' Manual," Dept. of Ocean Engineering, M.I.T.

8. Winterstein, S.R. (1988). "Nonlinear Vibration Models for Extremes and Fatigue," Journal of Engineering Mechanics, ASCE, Vol. 114, No. 10, pp. 1772-1790.

9. Winterstein, S.R., T.C. Ude, and G. Kleiven (1994). "Springing and Slow-Drift Responses: Predicted Extremes and Fatigue vs. Simulation," Proceedings, BOSS-94, Vol. 3, Massachusetts Institute of Technology, pp. 1-15.

10. Winterstein, S.R., A.K. Jha, and S. Kumar (1999). "Reliability of Floating Structures: Extreme Response and Load Factor Design," Journal of Water, Port, Coastal and Ocean Engineering, ASCE, Vol. 125, No. 4.

11. Winterstein, Steven R., and Sweetman, Bert (1999) "Air Gap Response of Floating Structures: Statistical Predictions vs Observed Behavior" Proceedings, OMAE 99, Paper No. OMAE 99-6042 


\begin{tabular}{|c|c|c|c|c|c|c|c|}
\hline Type of Run & Response & Mean (m) & Std. Dev (m) & Skewness & Kurtosis & $\begin{array}{l}\text { Median Maximum } \\
\text { (Minimum) }(\mathrm{m}) \\
\end{array}$ & Peak Factor \\
\hline Case 0 & TOTAL & 0.00 & 2.65 & 0.00 & 3.00 & $10.08(-10.08)$ & 3.8 \\
\hline \multirow[t]{2}{*}{ Case 1} & $1^{\text {st }}$-order & 0.00 & 2.65 & 0.00 & 3.00 & $10.08(-10.08)$ & \\
\hline & TOTAL & 0.00 & 2.70 & 0.20 & 3.06 & $11.17(-8.91)$ & 4.1 \\
\hline \multirow{2}{*}{ Case 2} & $1^{\text {st }}$-order & 0.00 & 3.25 & 0.00 & 3.00 & $12.08(-12.08)$ & \\
\hline & TOTAL & 0.00 & 3.27 & 0.09 & 3.04 & $12.11(-11.05)$ & 3.7 \\
\hline \multirow{2}{*}{ Case 3} & $1^{\text {st }}$-order & 0.00 & 3.25 & 0.00 & 3.00 & $12.08(-12.08)$ & \\
\hline & TOTAL & 0.00 & 3.50 & 0.54 & 4.12 & $19.23(-12.54)$ & 5.5 \\
\hline \multirow[t]{2}{*}{ Heave } & $1^{\text {st }}$-order & 0.00 & 0.78 & 0.00 & 3.00 & $2.81(-2.81)$ & \\
\hline & TOTAL & 0.07 & 0.79 & 0.21 & 3.13 & $3.34(-2.54)$ & 4.1 \\
\hline \multirow[t]{2}{*}{ Base } & $\mathbf{1}^{\text {st }}$-order & 0.00 & 2.52 & 0.00 & 3.00 & $9.44(-9.44)$ & \\
\hline & TOTAL & -0.07 & 2.85 & 0.50 & 4.55 & $17.18(-12.38)$ & 6.1 \\
\hline
\end{tabular}

Table 1. Response Statistics for Cases involving different Modeling Options 


\begin{tabular}{|c|c|c|c|c|c|c|c|c|}
\hline \multirow{2}{*}{ Field Point } & \multirow{2}{*}{$H_{\mathrm{s}}(\mathrm{m})$} & \multirow{2}{*}{$T_{\mathrm{p}}(\mathrm{s})$} & \multicolumn{2}{|c|}{ 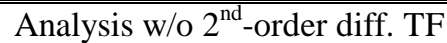 } & \multicolumn{2}{|c|}{ Analysis w/ $2^{\text {nd }}$-order diff. TF } & \multicolumn{2}{|c|}{ Wave Tank Tests } \\
\hline & & & $\sigma(\mathrm{m})$ & $\mathrm{PF}$ & $\sigma(\mathrm{m})$ & $\mathrm{PF}$ & $\sigma(\mathrm{m})$ & $\mathrm{PF}$ \\
\hline \multirow{4}{*}{$\begin{array}{l}\text { No. } 1 \\
\text { at center }\end{array}$} & 10.6 & 12.5 & 2.55 & 3.7 & 2.85 & 6.0 & - & - \\
\hline & 12.6 & 13.5 & 2.96 & 3.8 & 3.37 & 6.2 & 2.78 & 6.5 \\
\hline & 13.3 & 13.0 & 3.19 & 3.8 & 3.71 & 6.4 & - & - \\
\hline & 14.5 & 14.0 & 3.34 & 3.8 & 3.89 & 6.4 & 3.20 & 4.3 \\
\hline \multirow{4}{*}{$\begin{array}{c}\text { No. } 5 \\
\text { on diagonal }\end{array}$} & 10.6 & 12.5 & 3.16 & 4.1 & 3.26 & 5.3 & - & - \\
\hline & 12.6 & 13.5 & 3.53 & 4.1 & 3.68 & 5.6 & 3.56 & 4.6 \\
\hline & 13.3 & 13.0 & 3.87 & 4.2 & 4.05 & 5.7 & - & - \\
\hline & 14.5 & 14.0 & 3.93 & 4.2 & 4.15 & 5.8 & 4.10 & 5.1 \\
\hline \multirow{4}{*}{$\begin{array}{l}\text { No. } 6 \\
\text { in front of } \\
\text { column }\end{array}$} & 10.6 & 12.5 & 3.62 & 3.7 & 3.66 & 3.7 & - & - \\
\hline & 12.6 & 13.5 & 3.78 & 3.7 & 3.85 & 4.1 & 2.69 & 6.3 \\
\hline & 13.3 & 13.0 & 4.29 & 3.7 & 4.37 & 4.0 & - & - \\
\hline & 14.5 & 14.0 & 4.13 & 3.8 & 4.24 & 4.4 & 3.17 & 5.7 \\
\hline
\end{tabular}

Table 2. Predicted and Measured Response Statistics at Three Field Point Locations for Four Seastates. 


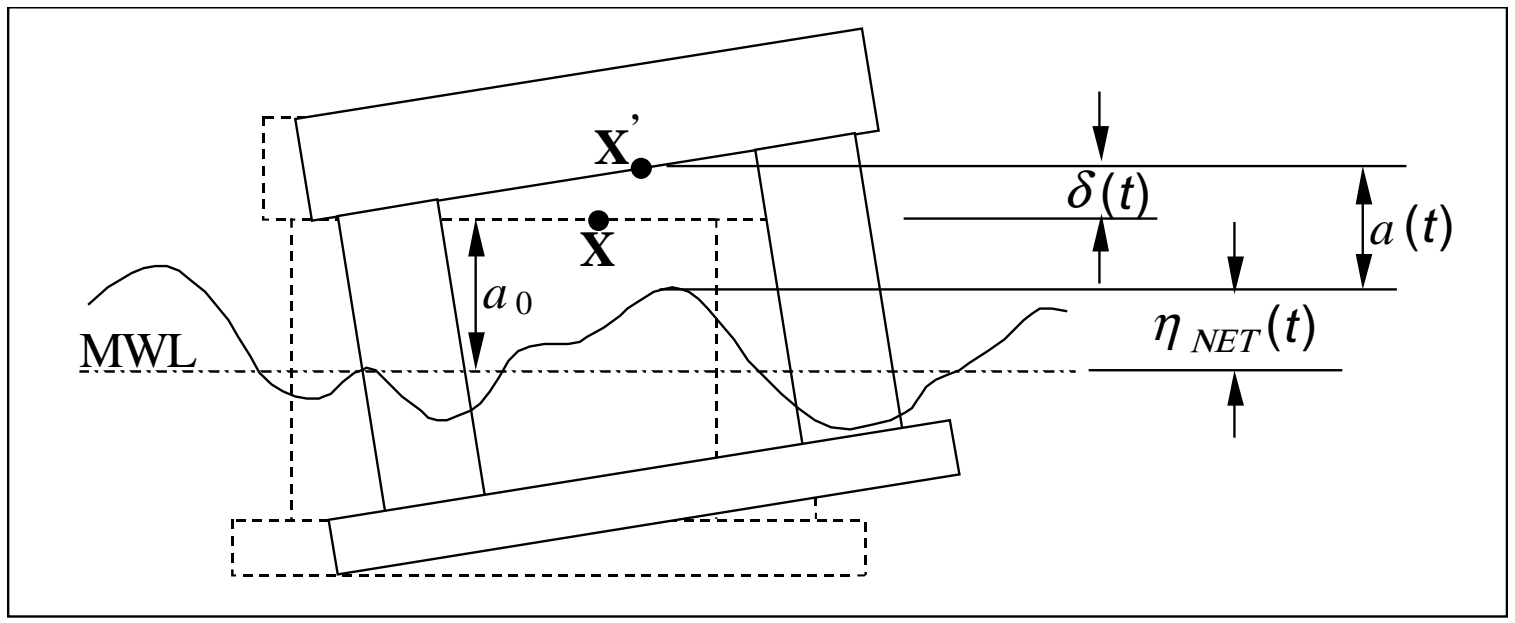

Figure 1. Definition of the instantaneous air gap, $a(t)$, in terms of the net wave elevation and the net vertical displacement. 


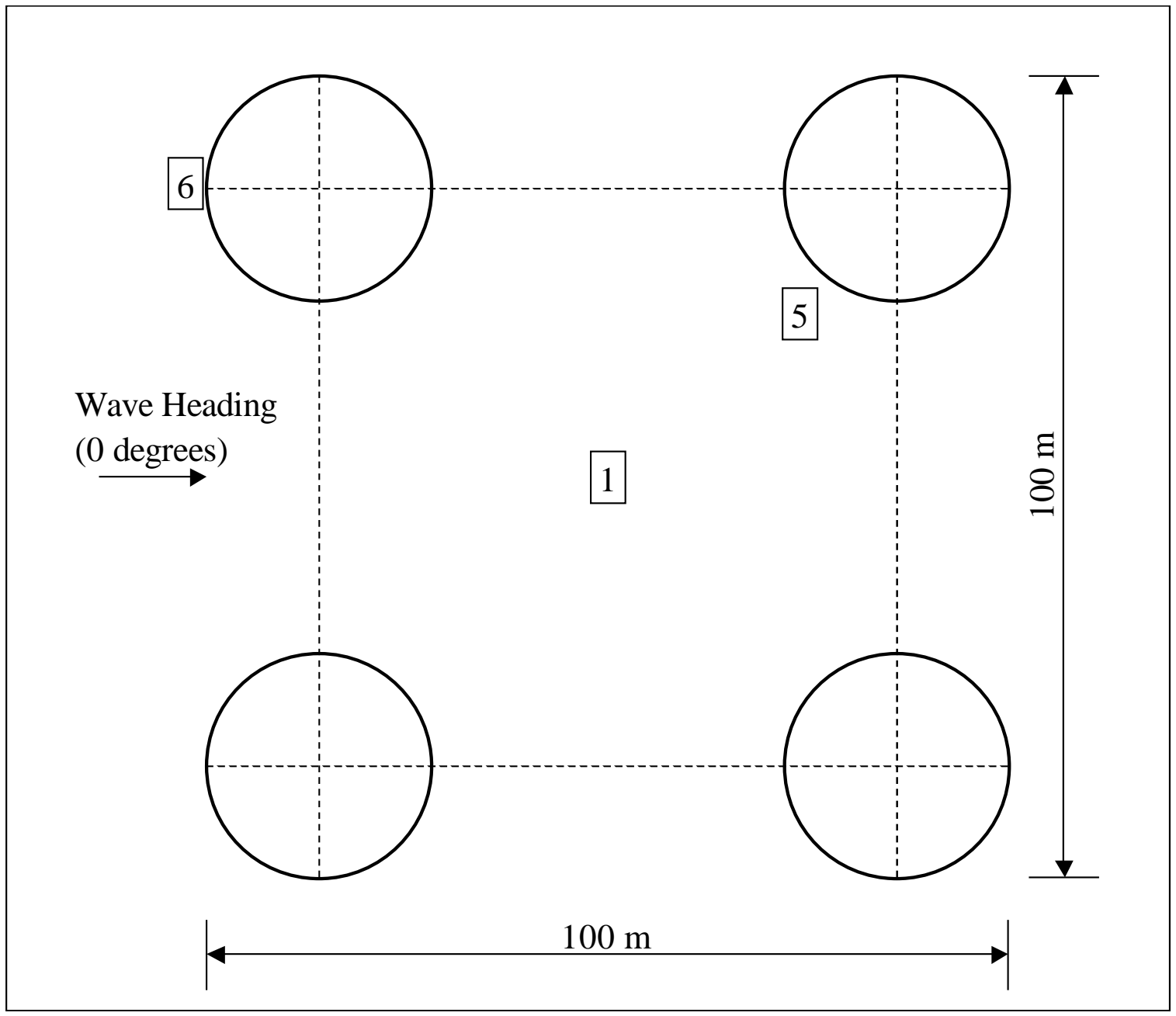

Figure 2. Plan view showing 4 columns of the Troll semi-submersible and the 3 field points (Nos. 1, 5, and 6) studied. 

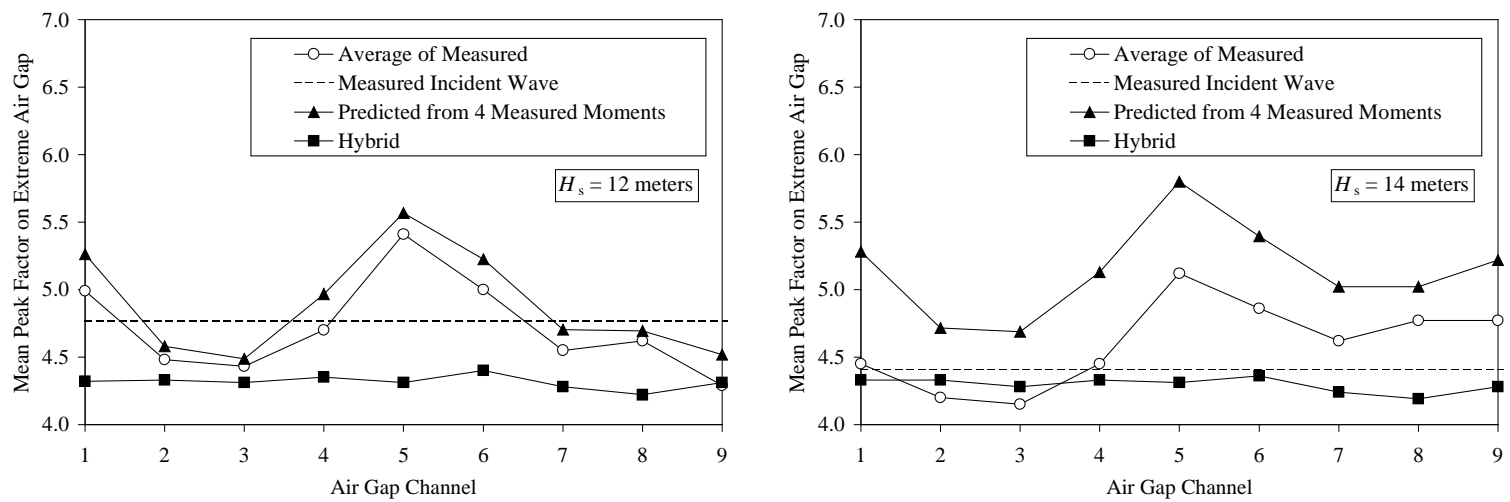

Figure 3. Veslefrikk air gap response (peak factor in 3 hours) - measurements versus analytical predictions at 9 different field points for two seastates, $H_{\mathrm{s}}=12$ and 14 meters. 